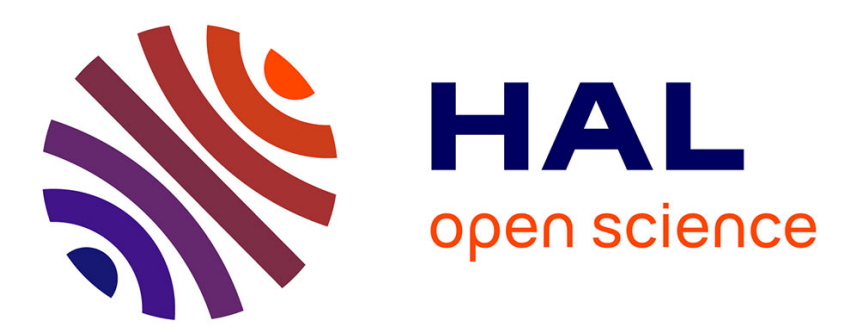

\title{
Kinematic sensitivity analysis of manipulators using a novel dimensionless index
}

Allaoua Brahmia, Ridha Kelaiaia, Olivier Company, Ahmed Chemori

\section{To cite this version:}

Allaoua Brahmia, Ridha Kelaiaia, Olivier Company, Ahmed Chemori. Kinematic sensitivity analysis of manipulators using a novel dimensionless index. Robotics and Autonomous Systems, 2022, 150, pp.104021. 10.1016/j.robot.2022.104021 . lirmm-03523086

\section{HAL Id: lirmm-03523086 https://hal-lirmm.ccsd.cnrs.fr/lirmm-03523086}

Submitted on 12 Jan 2022

HAL is a multi-disciplinary open access archive for the deposit and dissemination of scientific research documents, whether they are published or not. The documents may come from teaching and research institutions in France or abroad, or from public or private research centers.
L'archive ouverte pluridisciplinaire HAL, est destinée au dépôt et à la diffusion de documents scientifiques de niveau recherche, publiés ou non, émanant des établissements d'enseignement et de recherche français ou étrangers, des laboratoires publics ou privés. 


\title{
Kinematic sensitivity analysis of manipulators using a novel dimensionless index
}

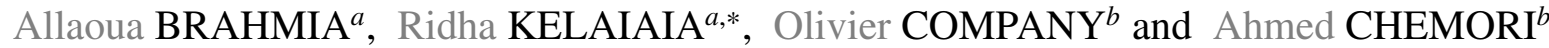 \\ ${ }^{a}$ Faculty of Technology, Université 20 août 1955-Skikda, \\ PB $N^{\circ} 26$ Route Elhadaik, Skikda 21000, Algeria \\ ${ }^{b}$ LIRMM, University of Montpellier, CNRS, Montpellier, France
}

\section{ARTICLE INFO}

\section{Keywords:}

Sensitivity analysis

New sensitivity index

PAR2 PKM

Position error modeling

Precision design

\begin{abstract}
A B S T R A C T
Geometric errors directly affect the position of the end-effector of a Parallel Kinematic Manipulator (PKM), thus reducing its positioning accuracy. However, the tasks that are performed by PKMs, such as high-precision machining using a kinematic model with nominal values, are affected by machine errors that are not taken into account. Therefore, it is important to make an accurate determination of a machine's error factors to obtain an accurate error model. Identifying the most crucial geometric errors and determining a method to control them is key in improving the accuracy of PKMs. To achieve this objective, a new method of sensitivity analysis, allowing the crucial geometric errors for parallel and serial manipulators to be identified, is proposed. A new dimensionless sensitivity index, based on the definition of a Local Sensitivity Index (LSI), is used to perform this analysis. The geometric error modeling is performed by deriving the position vector of the end-effector of the PKM. To test the efficiency of the proposed method, the main sources of PAR2 PKM errors are identified. The results show that $33.3 \%$ of the error components (main errors) from all error sources can be improved, to achieve a $51.8 \%$ improvement in the accuracy of the position error. These results indicate that the error sensitivity analysis method is quite effective, and can significantly contribute to improving the accuracy of a PKM.
\end{abstract}

\section{Introduction and related work}

Machine tools with a Cartesian structure suffer from the same defects as serial robots (low rigidity, low dynamic performance). In other words, feed speeds and accelerations are penalized by the high mass of the moveable elements, because each motorized axis supports the axis that follows it. The need to avoid these defects and to increase the working speeds of these tools has led researchers and industrialists to propose other machine architectures designed on the basis of parallel mechanisms, and these have made it possible to take a big step forward in the development of High-Speed Machining [1], which is now an industrial reality. PKMs [2] were targeted for the design and development of several prototypes in order to solve certain problems in the world of machining where the need for precision and high rigidity is crucial. These PKMs have attracted the attention of researchers and industrialists and present a feasible alternative to serial mechanisms, as they are characterized by low moving mass, high rigidity and ability to support a big load while working with good precision $[3,4,5,6,7,8]$.

In terms of accuracy, the positioning error of the end-effector of a serial manipulator is accumulated from the base through each link. On the other hand, the error factor of a parallel manipulator does not accumulate directly, but rather acts on average, increasing the accuracy. The configuration of PKMs with several Degrees-Of-Freedom (DOF) is more complex, because the end of each sub-chain converges at a single point. To ensure high motion accuracy in these complex configurations, PKMs require not only precise machining and assembly, but also high-performance control algorithms that incorporate error factors. For this reason, it is important to determine the cause of these errors in order to improve the accuracy of PKMs. Generally, there are two approaches to improving the accuracy of PKMs, namely precision design and kinematic calibration [9]. In precision design, error sensitivity analysis is used to discover the fundamental sources of error, and then more consideration is given to these sources of error in the manufacturing phase. In this way, a higher laying accuracy can be achieved [10,11, 12, 13]. Precision design is carried out at the design stage, without measuring instruments or procedures. Kinematic calibration is another method for improving

\footnotetext{
*Corresponding author

a.brahmia@univ-skikda.dz (A. BRAHMIA); r.kelaiaia@univ-skikda.dz (R. KELAIAIA); company@lirmm.fr (O.

COMPANY); Ahmed. chemori@lirmm.fr (A. CHEMORI)

$\operatorname{ORCID}(\mathrm{s})$ :
} 
PKM accuracy. In this method, measuring devices to inspect the position errors of the end-effector are used. For reasons of expense and convenience, precision design is widely accepted by manufacturers of manipulators, while kinematic calibration is generally adopted by users of these manipulators [12]. Regardless of the type of method used, the modeling and sensitivity analysis of geometric errors remain unavoidable. The identification of the main sources of error always remains a crucial point.

The main objective of sensitivity analysis is to identify the main sources of error by using an error sensitivity index. Greater consideration must be given to these main sources of error, in order to obtain higher accuracy in the manipulator positioning. It should be noted that the sensitivity analysis methods vary depending to the methods used for the error modeling. A seminal contribution was made by Lee et al. [14], who applied a simplified error model on a tripyramid parallel translation manipulator with three DOFs. Kinematic error sources were identified using the linearized homogeneous transformation matrix (HTM) and the Denevit-Hartenberg (DH) parameters. Error sources affecting the accuracy of the platform position were selected using a sensitivity analysis method. The results of the simulation of the calibration showed that the accuracy of the model could be improved by $70 \%$ (from 0.85 to $0.26 \mathrm{~mm}$ ). The advantage of this method lies in its application to parallel and serial manipulators. In addition, the error model can be used to improve the accuracy during the manufacture of the manipulator. A comprehensive description can be found in [11]. These authors used a multi-body system (MBS) error modeling method to construct the error mapping between the error sources and the cutting tool position error for a five-axis machine tool. New indices were used, such as General Local Sensitivity Indices (GLSI), General Global Sensitivity Indices (GGSI) and Global Sensitivity Index Fluctuation Indices (GGSFI), for the error sensitivity analysis. The results indicated that an improvement in the accuracy of the cutting tool could be achieved with less improvement of the error components. Using the same manipulator, Guo et al. [10] performed a global quantitative sensitivity analysis of each geometric error affecting the position and orientation of the machine. Their results showed that the geometric errors of the rotary axes were the dominant sensitivity factors, and that the compensation of the geometric errors was improved by $56.22 \%$. A series of recent studies have used Local Sensitivity Indices (LSIs) $[15,16,11,17]$ to analyze the sensitivity of a manipulator to a specific pose (posture). These studies have calculated the influence of dimensional variations on the position of the manipulator end-effector. These indices were tested on an Orthoglide Parallel Kinematic Machine with three DOFs [16]. This has also been explored in other studies. In [16], the authors calculated the LSIs for a machine tool in an experimental test, while in [15] the authors calculated these indices on a 3-PRS (P: prismatic joint, R: revolute joint and S: ball-and-socket joint) parallel kinematic spindle platform of a serial-parallel machine tool.

Some researchers have suggested other approaches to perform the sensitivity analysis. For example, Caro et al. [18] presented an error index for the position and orientation of a 3-RPR planar parallel manipulator, and proposed a method to evaluate the performance of the manipulator in terms of dexterity, workspace, and sensitivity. Tannous et al. [19] used an interval linearization method to analyze the sensitivity of three types of PKM to variations in the geometric parameters. Palpacelli et al. [20] developed a kinematic error model for a mini spherical manipulator. A sensitivity analysis of all the geometric parameters was performed. After validation of their model, a calibration of the manipulator was performed. To calibrate a six DOFs industrial manipulator, Qi et al. [21] used a mathematical model based on the Jacobian matrix.

In order to discover the significance of the influence of the physical errors on the end-effector error of a manipulator, Zhang et al. [22] carried out an error analysis based on the DH matrix. Their method can be used to estimate the error calibration of other manipulators. Li et al. [23] established a model for the representation of position-dependent geometric errors for linear axes based on moving least squares and the Chebyshev polynomial method. The position accuracy of the motion axis was improved by $90 \%$ after error compensation.

Chanal and all. [24] proposed a new method for quantifying design constraints. It consists of the analysis of the sensitivity of the matrix of the inverse kinematic model to determine the different relations which must be verified in order to discount pose defects which cannot be compensated.

Sensitivity indices can be defined in a statistical sense. There have been numerous studies to investigate this context. Guo et al. [25] proposed an approach to improve the accuracy of five-axis machine tools. The Extended Fourier Amplitude Sensitivity Test (EFAST) method was used to analyze the overall sensitivity of the geometric errors. The results showed that the accuracy of the measurement paths was significantly improved, reaching $73.7 \% \%$ after error compensation. Another method was proposed by $\mathrm{Ni}$ et al. [26] to improve the accuracy of a parallel manipulator with full-circle rotation. Sensitivity analysis was performed on the sources of the geometric errors using a global sensitivity evaluation index. A probability model was used for this analysis. The results of this analysis provided a theoretical basis for the allocation of tolerances to parts of the mechanical design. To study the sensitivity of a PKM with SCARA 
motions, Yuzhen et al. [27] carried out a sensitivity analysis based on an error model and sensitivity indices defined in the statistical sense. Some atlases are depicted to express each geometric error's influence on the platform pose errors. From these atlases, the geometric errors with greater impact on the accuracy of the platform are identified, and the areas where the pose errors of the platform are extremely sensitive to the geometric errors are also highlighted. Cheng et al. [28] used an analytical approach to identify critical geometric errors of multi-axis machine tools. The stochastic characteristics of geometric errors were taken into account, and the Sobol global sensitivity analysis method was introduced to identify these errors. The sensitivity and kinematic calibration of a 3-UCR symmetrical parallel robot leg were analyzed by Cheng et al. [29]. Based on the complete differential-coefficient theory, a model of the pose errors based on the Rodrigues Parameters [30] was established. Tian et al. [31] studied the influence, in the statistical sense, of geometric errors on the volumetric accuracy of a five-axis machine tool. This method made it possible to calculate the compensable and the non-compensable error sources of the machine tool. The author did not judge the effectiveness of the method by indicating the rate of improvement of the position error after determination of the main sources of error.

The difficulties that can be overcome by this work are:

- Determine the vital errors of parallel robots: knowledge of the vital errors makes it possible to obtain interesting information on the sensitivity of the robots in relation to the variations of the geometric parameters;

- Improve the accuracy of parallel robots from the design phase: vital errors can be used by the design precision method to verify the accuracy of parallel robots from the design phase;

- Carrying out a robust design of the parallel robots: in [32], vital errors are used to carry out a robust design of the parallel robots.

Therefore, the most challenge studied in this paper is to propose and develop a dimensionless sensitivity index, and then find the vital errors using this index.

It should be noted that for the majority of the work cited in $[14,15,16,17,18,19,20,21,22,23,24,25,26,27,28$, 29, 30], most sensitivity indices are obtained by:

- Calculation of the LSI $[15,16,17,20]$ : Where, the LSIs are obtained by derivation of the elements of the Jacobian matrix which links the variation of the position error to the variations of the geometric parameters of the robot. However, the determination of this Jacobian matrix is sometimes very difficult, especially for robots with complex structures;

- Geometric analysis [11, 12,14]: In this type of analysis, sensitivity indices are generally simplified by ignoring the terms of expression of these indices. Consequently, the sensitivity analysis may suffer from the accuracy problem;

- Statistical analysis [10, 25, 26, 27, 28, 29]: The calculation of sensitivity indices is affected by the approximation aspect (the average, standard deviation, etc.). In this case, the use of this type of indices affects the accuracy of the robot sensitivity analysis.

To overcome the problems related to these indices, a dimensionless sensitivity index has been proposed. Its calculation is based on knowledge of LSI. It is note that the LSIs are easily extracted from the Jacobian matrix which links the vector of the position error variations to the variations of the geometric parameters of the robot. In addition, the proposed sensitivity index does not require the knowledge of tolerance intervals during sensitivity analysis, and it can be used for all types of robots (both serial and parallel). The main contributions of this paper are:

i) A novel dimensionless sensitivity index, based on the definition of the LSI, is proposed to perform a sensitivity analysis of the manipulator;

ii) A methodology for the error sensitivity analysis of PAR2 PKM is proposed, in order to determine the main geometric errors;

iii) The linear and angular position geometric errors of the PAR2 PKM are identified using the proposed index;

iv) Calculation of the position error under the precision design based on error sensitivity analysis. 
The remainder of this paper is organized as follows. In section 2, the sensitivity indices for the sensitivity analysis method are defined. In section 3, a geometric description of the PAR2 manipulator is introduced. A sensitivity analysis of the errors of the manipulator is performed using the proposed sensitivity indices. The sensitivity analysis of the position error is verified. Finally, the article is summarized in section 4.

\section{Definition of sensitivity indices}

In order to evaluate the influence of variations in the dimensional tolerances of the manipulator components on the position error of the end-effector, a sensitivity analysis of these variations is essential. The definition of sensitivity indices is the major problem encountered in this type of analysis.

\subsection{Definition of LSI and GSI indices}

The LSI and GSI (Global Sensitivity Index) sensitivity indices are obtained mathematically by partial differentiation of the expression containing the geometric parameters. It should be noted that the LSI depends significantly on the configuration and geometric parameters of the PKM. It evolves within the workspace, so it is a local property. To characterize it over the whole space accessible by the PKM, we use the global sensitivity index GSI, which is defined as the average of the local sensitivities over the reachable space [11]. In order to illustrate these definitions, we assume the kinematic chain consisting of $n$ segments shown in Figure 1. The expression for the position vector $E$ in space is

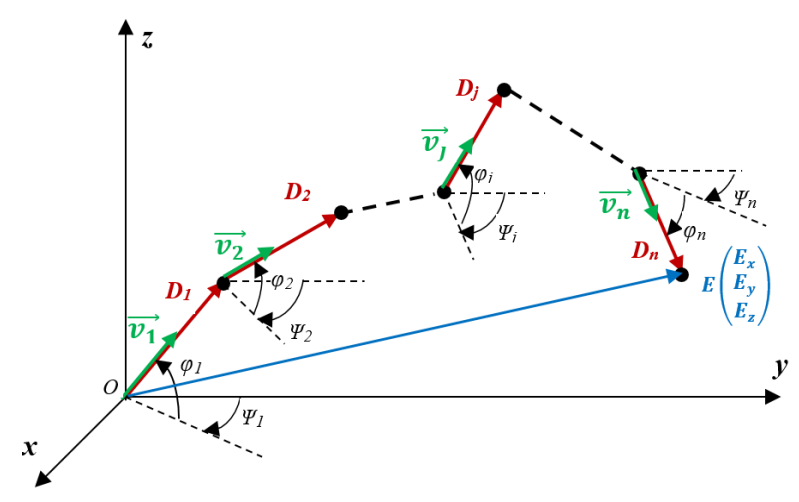

Figure 1: Parametrization of the kinematic chain.

given as follows:

$$
\overrightarrow{O E}=\left(\begin{array}{c}
E_{x} \vec{x} \\
E_{y} \vec{y} \\
E_{z} \vec{z}
\end{array}\right)=\sum_{j=1}^{n} D_{j} \overrightarrow{v_{j}}
$$

where:

$D_{j}$ : Length of the $j^{\text {th }}$ dimension.

$\overrightarrow{v_{j}}$ : Unit vector $\overrightarrow{D_{j}} /\left\|\overrightarrow{D_{j}}\right\|_{2}$.

After differentiation of equation (1), we obtain:

$$
\delta \overrightarrow{O E}=\sum_{j=1}^{n}\left(\delta D_{j} \overrightarrow{v_{j}}+D_{j} \delta \overrightarrow{v_{j}}\right)
$$

where $\delta \overrightarrow{O E}$ is the variation of the position error of the manipulator. Similarly, $\delta D_{j}$ denotes the variation of $D_{j}$. The expression $\overrightarrow{v_{j}}$ contains the angular variations $\delta \varphi_{j}$ and $\delta \psi_{j}$ which represents the variations of $\varphi_{j}$ and $\psi_{j}$.

Equation (2) can be expressed in matrix form as follows:

$$
\delta O E=J \delta P
$$


Tell that:

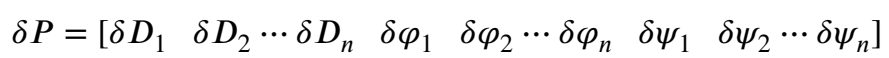

where $J$ is the sensitivity Jacobian matrix.

The partial derivative of equation (1) with respect to each dimensional variation $\delta P_{j}$, is given by the following expression:

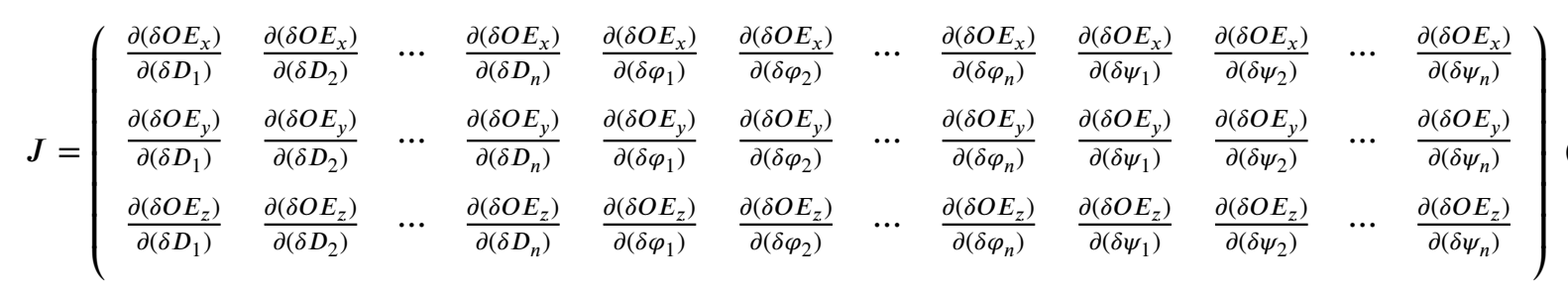

The elements of the $J$ matrix are none other than the $L S I$ of the $\delta P_{j}$ dimensional variations, i.e.:

For $j=1, \cdots, n$

$$
\left\{\begin{array}{l}
\frac{\partial\left(\delta O E_{x}\right)}{\partial\left(\delta P_{j}\right)}=L S I_{x}\left(\delta P_{j}\right) \\
\frac{\partial\left(\delta O E_{y}\right)}{\partial\left(\delta P_{j}\right)}=L S I_{y}\left(\delta P_{j}\right) \\
\frac{\partial\left(\delta O E_{z}\right)}{\partial\left(\delta P_{j}\right)}=L S I_{z}\left(\delta P_{j}\right)
\end{array}\right.
$$

the expression of $J$ then becomes:

$$
J=\left(\begin{array}{cccc}
L S I_{x}\left(\delta P_{1}\right) & L S I_{x}\left(\delta P_{2}\right) & \cdots & L S I_{x}\left(\delta P_{n}\right) \\
L S I_{y}\left(\delta P_{1}\right) & L S I_{y}\left(\delta P_{2}\right) & \cdots & L S I_{y}\left(\delta P_{n}\right) \\
L S I_{z}\left(\delta P_{1}\right) & L S I_{z}\left(\delta P_{2}\right) & \cdots & L S I_{z}\left(\delta P_{n}\right)
\end{array}\right)
$$

$L S I_{x}\left(\delta P_{j}\right)$ is the local sensitivity index on the $x$ direction linked to the parameter $\delta P_{j}$, while $L S I_{y}\left(\delta P_{j}\right)$ is the local sensitivity index on the $y$ direction linked to the parameter $\delta P_{j}$ and $L S I_{z}\left(\delta P_{j}\right)$ is the local sensitivity index on the $z$ direction linked to the parameter $\delta P_{j}$.

Usually, we are interested in the influence of dimensional variations in the workspace more than in a specific pose. However, the calculation of the GSIs is performed by an integration of the LSIs. Hence the definition of GSI is given as follows:

$$
G S I=\frac{\int_{w} L S I d w}{W}
$$

where $W$ is the workspace.

\subsection{Definition of the sensitivity coefficient $S e$}

Sensitivity analysis is used to analyze the variation of the position error with respect to the dimensional variations of the elements of the PKM structure. Each kinematic parameter can therefore be analyzed in terms of how it affects the accuracy of the manipulator. However, the sensitivity coefficient is defined as a non-dimensional number [33]. The expression of the sensitivity coefficient is given by the following relation [14]:

$$
S e_{j}=\frac{\left\|\overrightarrow{\delta E\left(\delta P_{j}\right)}\right\|_{2}}{\left\|\delta \overrightarrow{O E\left(\sum_{i=1}^{n} \delta P_{i}\right)}\right\|_{2}} \quad j=1, \cdots, n
$$

where:

$\delta \overrightarrow{O E\left(\delta P_{j}\right)}$ is the position error due to the dimensional variation $\delta P_{j}$, and $\delta \overrightarrow{O E\left(\sum_{i=1}^{n} \delta P_{i}\right)}$ is the position error due to the variation of all the dimensional parameters $\delta P_{j}$. 


\subsection{New sensitivity index}

Sensitivity analysis aims to find the main sources of error, in order to compensate for the error or to use precision design, throughout the workspace. We can define the error sources at the same level to examine which can cause a large error in the position [11]. However, all the dimensional variations can be configured as a single unit in their own category, namely:

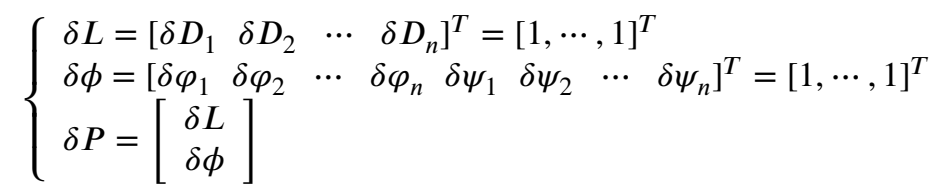

Hence:

$\delta L$ is the linear error vector, and $\delta \phi$ is the angular error vector.

According to equations (3), (7) and (10), the expressions of the sensitivity coefficient $S e_{j}$ can be written in the following form:

$$
S e_{j}=\frac{\sqrt{\left(L S I_{x}\left(\delta P_{j}\right)\right)^{2}+\left(L S I_{y}\left(\delta P_{j}\right)\right)^{2}+\left(L S I_{z}\left(\delta P_{j}\right)\right)^{2}}}{\sqrt{\left(\sum_{i=1}^{n} L S I_{x}\left(\delta P_{i}\right)\right)^{2}+\left(\sum_{i=1}^{n} L S I_{y}\left(\delta P_{i}\right)\right)^{2}+\left(\sum_{i=1}^{n} L S I_{z}\left(\delta P_{i}\right)\right)^{2}}}
$$

After substituting $\delta L_{j}$ and $\delta \phi_{j}$ in equation (11), three cases are distinguished:

\section{Case 1:}

If we consider only the variation of the dimensional parameter $\delta D_{j}$, equation (2) becomes:

$$
\delta \overrightarrow{O E\left(\delta D_{j}\right)}=\delta D_{j} \overrightarrow{v_{j}}
$$

Knowing that:

$$
\overrightarrow{v_{j}}=\cos \left(\varphi_{j}\right) \sin \left(\psi_{j}\right) \vec{x}+\cos \left(\varphi_{j}\right) \cos \left(\psi_{j}\right) \vec{y}+\sin \left(\varphi_{j}\right) \vec{z}
$$

and

$\delta D_{j}=1$ (according to equation (10)). Therefore, the norm of the variation of the position error is given by:

$$
\left\|\overrightarrow{\delta E\left(\delta D_{j}\right)}\right\|_{2}=\sqrt{\left(\cos \left(\varphi_{j}\right) \sin \left(\psi_{j}\right)\right)^{2}+\left(\cos \left(\varphi_{j}\right) \cos \left(\psi_{j}\right)\right)^{2}+\left(\sin \left(\varphi_{j}\right)\right)^{2}}=1
$$

Then, equation (11) becomes:

$$
S e_{j}=\frac{1}{\sqrt{\left(\sum_{i=1}^{n} L S I_{x}\left(\delta P_{i}\right)\right)^{2}+\left(\sum_{i=1}^{n} L S I_{y}\left(\delta P_{i}\right)\right)^{2}+\left(\sum_{i=1}^{n} L S I_{z}\left(\delta P_{i}\right)\right)^{2}}}
$$

\section{Case 2:}

If we consider only the variation of the angular parameter $\delta \varphi_{j}$, equation (2) becomes:

$$
\delta \overrightarrow{O E\left(\delta \varphi_{j}\right)}=D_{j} \delta \overrightarrow{v_{j}}
$$

Knowing that:

$$
\delta \overrightarrow{v_{j}}=-\delta \varphi_{j} \sin \left(\varphi_{j}\right) \sin \left(\psi_{j}\right) \vec{x}-\delta \varphi_{j} \sin \left(\varphi_{j}\right) \cos \left(\psi_{j}\right) \vec{y}+\delta \varphi_{j} \cos \left(\varphi_{j}\right) \vec{z}
$$

and

$\delta \varphi_{j}=1$ (according to equation (10)). Therefore, the norm of the variation of the position error is given by:

$$
\left.\left\|\overrightarrow{O E\left(\delta \varphi_{j}\right)}\right\|_{2}=\sqrt{D_{j}^{2}\left[\left(-\sin \left(\varphi_{j}\right) \sin \left(\psi_{j}\right)\right)^{2}+\left(-\sin \left(\varphi_{j}\right) \cos \left(\psi_{j}\right)\right)^{2}+\left(\cos \left(\varphi_{j}\right)\right)^{2}\right.}\right]=D_{j}
$$


Equation (11) then becomes:

$$
S e_{j}=\frac{D_{j}}{\sqrt{\left(\sum_{i=1}^{n} L S I_{x}\left(\delta P_{i}\right)\right)^{2}+\left(\sum_{i=1}^{n} L S I_{y}\left(\delta P_{i}\right)\right)^{2}+\left(\sum_{i=1}^{n} L S I_{z}\left(\delta P_{i}\right)\right)^{2}}}
$$

\section{Case 3:}

If we consider only the variation of the angular parameter $\delta \psi_{j}$, the expression for the sensitivity coefficient $S e_{j}$ becomes:

$$
S e_{j}=\frac{D_{j} \cos \left(\varphi_{j}\right)}{\sqrt{\left(\sum_{i=1}^{n} L S I_{x}\left(\delta P_{i}\right)\right)^{2}+\left(\sum_{i=1}^{n} L S I_{y}\left(\delta P_{i}\right)\right)^{2}+\left(\sum_{i=1}^{n} L S I_{z}\left(\delta P_{i}\right)\right)^{2}}}
$$

From the analysis of the preceding cases, we notice that if the variations are only dimensional (case 1), the value of the sensitivity coefficient $S e_{j}$ remains unchanged for all $\delta D_{j}$, and it depends only on the sum of $L S I$. Indeed, to distinguish the sensitivity coefficient related to each error from the other coefficients, we propose to make a transformation of the relation (11) by the following change:

$$
\left\{\begin{array}{l}
S I_{x}\left(\delta P_{j}\right)=S e_{j} \frac{\partial O E_{x}}{\partial P_{j}} \frac{\partial P_{j}}{\partial O E_{x}} \\
S I_{y}\left(\delta P_{j}\right)=S e_{j} \frac{\partial E_{y}}{\partial P_{j}} \frac{\partial P_{j}}{\partial O E_{y}} \\
S I_{z}\left(\delta P_{j}\right)=S e_{j} \frac{\partial O E_{z}}{\partial P_{j}} \frac{\partial P_{j}}{\partial O E_{z}}
\end{array}\right.
$$

$\frac{\partial O E_{x}}{\partial P_{j}}$ are none other than the $L S I_{x}\left(\delta P_{j}\right)$, and $\frac{\partial P_{j}}{\partial O E_{x}}=1$ because the variation $\delta P_{j}$ is always equal to 1 (equation (10)). We do the same thing for $\frac{\partial O E_{y}}{\partial P_{j}}, \frac{\partial P_{j}}{\partial O E_{y}}$ and $\frac{\partial O E_{z}}{\partial P_{j}}, \frac{\partial P_{j}}{\partial O E_{z}}$. The system of equations (21) the becomes:

For $j=1, \cdots, n$

$$
\left\{\begin{array}{l}
S I_{x}\left(\delta P_{j}\right)=S e_{j} L S I_{x}\left(\delta P_{j}\right)=\frac{L S I_{x}\left(\delta P_{j}\right) \sqrt{\left(L S I_{x}\left(\delta P_{j}\right)\right)^{2}+\left(L S I_{y}\left(\delta P_{j}\right)\right)^{2}+\left(L S I_{z}\left(\delta P_{j}\right)\right)^{2}}}{\sqrt{\left(\sum_{i=1}^{n} L S I_{x}\left(\delta P_{i}\right)\right)^{2}+\left(\sum_{i=1}^{n} L S I_{y}\left(\delta P_{i}\right)\right)^{2}+\left(\sum_{i=1}^{n} L S I_{z}\left(\delta P_{i}\right)\right)^{2}}} \\
S I_{y}\left(\delta P_{j}\right)=S e_{j} L S I_{y}\left(\delta P_{j}\right)=\frac{L S I_{y}\left(\delta P_{j}\right) \sqrt{\left(L S I_{x}\left(\delta P_{j}\right)\right)^{2}+\left(L S I_{y}\left(\delta P_{j}\right)\right)^{2}+\left(L S I_{z}\left(\delta P_{j}\right)\right)^{2}}}{\sqrt{\left(\sum_{i=1}^{n} L S I_{x}\left(\delta P_{i}\right)\right)^{2}+\left(\sum_{i=1}^{n} L S I_{y}\left(\delta P_{i}\right)\right)^{2}+\left(\sum_{i=1}^{n} L S I_{z}\left(\delta P_{i}\right)\right)^{2}}} \\
S I_{z}\left(\delta P_{j}\right)=S e_{j} L S I_{z}\left(\delta P_{j}\right)=\frac{L S I_{z}\left(\delta P_{j}\right) \sqrt{\left(L S I_{x}\left(\delta P_{j}\right)\right)^{2}+\left(L S I_{y}\left(\delta P_{j}\right)\right)^{2}+\left(L S I_{z}\left(\delta P_{j}\right)\right)^{2}}}{\sqrt{\left(\sum_{i=1}^{n} L S I_{x}\left(\delta P_{i}\right)\right)^{2}+\left(\sum_{i=1}^{n} L S I_{y}\left(\delta P_{i}\right)\right)^{2}+\left(\sum_{i=1}^{n} L S I_{z}\left(\delta P_{i}\right)\right)^{2}}}
\end{array}\right.
$$

Hence: $S I_{x}\left(\delta P_{j}\right), S I_{y}\left(\delta P_{j}\right)$ and $S I_{z}\left(\delta P_{j}\right)$ are the new sensitivity indices of the components of the dimensional parameter variation $\left(\delta P_{j}\right)$ in the $x, y$ and $z$ directions respectively. They represent the sensitivity of the manipulator to variations in the dimensional parameters for each posture.

To characterize this over the whole space accessible by the manipulator, we define the global sensitivity index $(G)$ by the following expression:

For $j=1, \cdots, n$

$$
\left\{\begin{array}{l}
G_{x}\left(\delta P_{j}\right)=\frac{\int_{w} S I_{x}\left(\delta P_{j}\right) d w}{W} \\
G_{y}\left(\delta P_{j}\right)=\frac{\int_{w} S I_{y}\left(\delta P_{j}\right) d w}{W} \\
G_{z}\left(\delta P_{j}\right)=\frac{\int_{w} S I_{z}\left(\delta P_{j}\right) d w}{W}
\end{array}\right.
$$

Hence $G_{x}\left(\delta P_{j}\right), G_{y}\left(\delta P_{j}\right)$ and $G_{z}\left(\delta P_{j}\right)$ are the $G S I$ of the components of the dimensional parameter variation $\left(\delta P_{j}\right)$ on the position error in the $x, y$ and $z$ directions, respectively. 


\subsection{Definition of the VSI of the proposed SI index}

The proposed index $G$ can become a standard for selecting certain errors that have a greater relative influence on the end-effector position error. However, in some cases, an error has a small average influence on the end-effector position error but fluctuates considerably in the workspace. For this type of error, we will have a smaller $G$, but the accuracy can still be worse for some poses. Variance is widely used in industrial production to indicate the fluctuation of samples, and it can also be used to demonstrate the fluctuation of the influence of an error [12]. Like the definition of variance, the variance sensitivity index (VSI) of an error on the end-effector position error vector can be expressed by the following relationship:

For $j=1, \cdots, n$

$$
\left\{\begin{array}{l}
V G_{x}\left(\delta P_{j}\right)=\frac{\int_{w}\left(S I_{x}\left(\delta P_{j}\right)\right)^{2} d w}{W}-\left(G_{x}\left(\delta P_{j}\right)\right)^{2} \\
V G_{y}\left(\delta P_{j}\right)=\frac{\int_{w}\left(S I_{y}\left(\delta P_{j}\right)\right)^{2} d w}{W}-\left(G_{y}\left(\delta P_{j}\right)\right)^{2} \\
V G_{z}\left(\delta P_{j}\right)=\frac{\int_{w}\left(S I_{z}\left(\delta P_{j}\right)\right)^{2} d w}{W}-\left(G_{z}\left(\delta P_{j}\right)\right)^{2}
\end{array}\right.
$$

Hence $V G_{x}\left(\delta P_{j}\right), V G_{y}\left(\delta P_{j}\right)$, and $V G_{z}\left(\delta P_{j}\right)$ are the VSI of the component of the dimensional parameter variation $\left(\delta P_{j}\right)$ on the position errors in the $x, y$ and $z$ directions, respectively.

\section{Case study: PAR2 PKM}

PAR2 is a PKM with two DOFs composed of two motorized (active) kinematic chains and two other passive chains built in the transverse plane to stiffen the structure of the manipulator (Figure 2); this allows two translations in the vertical plane, while guaranteeing good stiffness in the transverse axis. The passive arms are used to prevent, as far as possible, perpendicular movements (out of the $x z$ plane; see Figure 3). For more details, the reader can refer to $[34,35,36]$ concerning the mechanical design and the control of this robot. In this work, a new index valid for 3D manipulators without effector orientation is proposed. This index has been subsequently applied to the PAR2 parallel robot with two DOFs acting in the $x z$ plane. Figure 1 shows a general case of a 3D position vector. For this figure to be consistent with the PAR2 robot, the angle $\psi_{i}$ must be equal to zero. To simplify the realization of the architecture's,

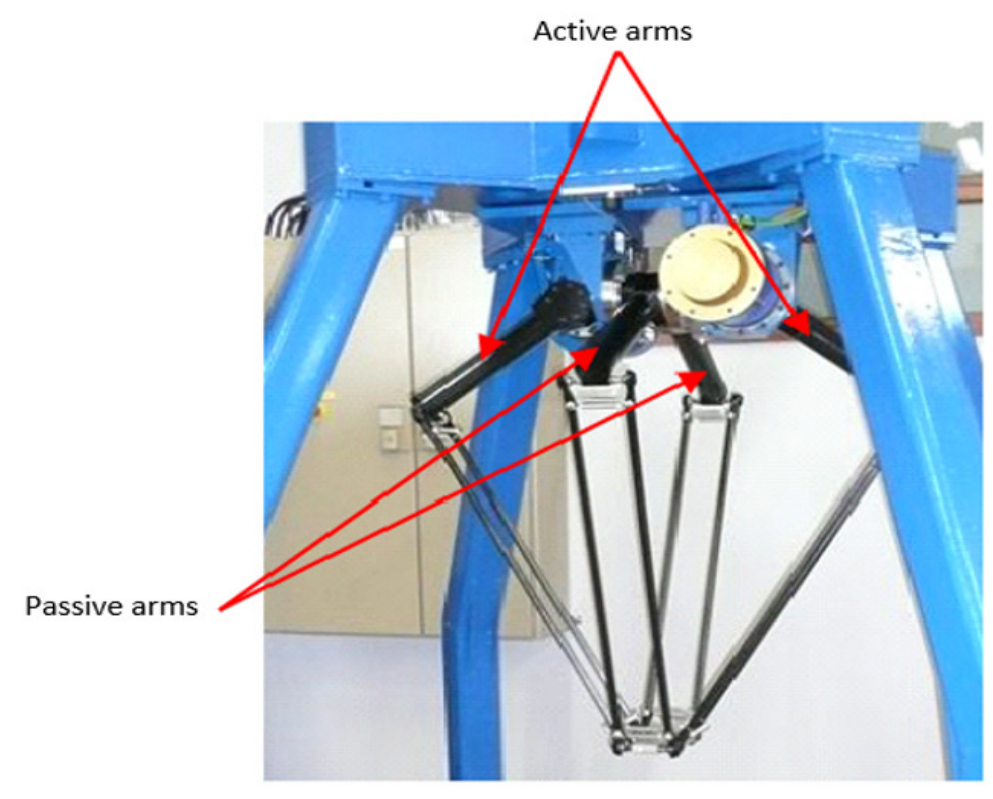

Figure 2: View of the PAR2 parallel manipulator [34]. 


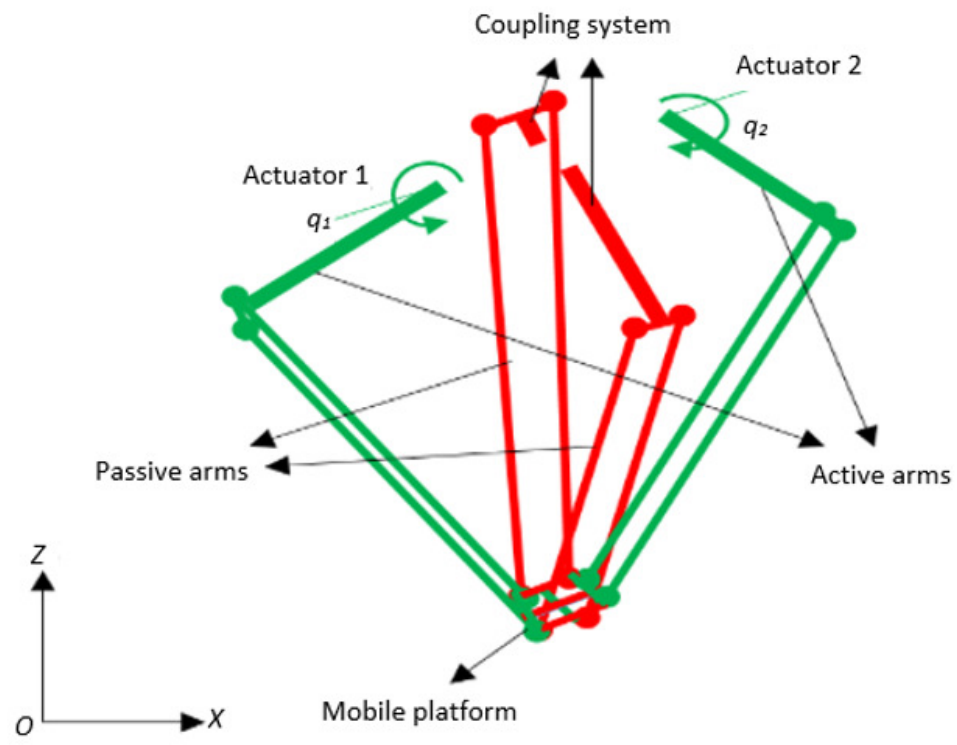

Figure 3: Schematic CAD view of the PAR2 PKM.

we treat the two branches $P_{1} A_{1} B_{1}$ and $P_{2} A_{2} B_{2}$ as identical. Therefore, the geometric parameters of this manipulator (Figure 4) are:

$L a_{i}$ : Arm length $P_{i} A_{i}(i \in 1,2)$

$L b_{i}$ : Arm length $A_{i} B_{i}$

$d_{i}$ : Distance between points $B_{i}$ and $C$ on the mobile platform

$D_{i}$ : Distance between points $P_{i}$ and $O$ on the base

\subsection{Modeling the position error of the PAR2 PKM}

From the closed kinematic chains $O-P_{i}-A_{i}-B_{i}-C-O, i=1, \cdots, 2$ shown in Figure 4, the position vector of point $\mathrm{C}$ can be expressed as follows:

$$
\overrightarrow{O C}=\left[\begin{array}{c}
C_{x} \vec{x} \\
C_{z} \vec{y}
\end{array}\right]=D_{i} \overrightarrow{h_{i}}+L a_{i} \overrightarrow{u_{i}}+L b_{i} \overrightarrow{v_{i}}+d_{i} \overrightarrow{g_{i}}
$$

where:

$\overrightarrow{h_{i}}$ is the unit vector $\frac{\overrightarrow{O P_{i}}}{\| \overrightarrow{O P}_{i}}$,

$\overrightarrow{u_{i}}$ is the unit vector $\frac{\overrightarrow{P_{i} A_{i}}}{\left\|\overrightarrow{P_{i} A_{i}}\right\|_{2}}$,

$\overrightarrow{v_{i}}$ is the unit vector $\frac{\overrightarrow{A_{i} B_{i}}}{\left\|\overrightarrow{A_{i} B_{i}}\right\|_{2}}$,

and $\overrightarrow{g_{i}}$ is the unit vector $\frac{\overrightarrow{B_{i} C}}{\left\|\overrightarrow{B_{i} C}\right\|_{2}}$.

$h_{1}=\left[\begin{array}{l}1 \\ 0\end{array}\right], \quad h_{2}=\left[\begin{array}{c}-1 \\ 0\end{array}\right], \quad u_{1}=\left[\begin{array}{c}\cos \left(\varphi_{11}\right) \\ -\sin \left(\varphi_{11}\right)\end{array}\right], \quad u_{2}=\left[\begin{array}{c}-\cos \left(\varphi_{12}\right) \\ -\sin \left(\varphi_{12}\right)\end{array}\right], \quad v_{1}=\left[\begin{array}{l}-\cos \left(\varphi_{21}\right) \\ -\sin \left(\varphi_{21}\right)\end{array}\right]$,

$v_{2}=\left[\begin{array}{c}\cos \left(\varphi_{22}\right) \\ -\sin \left(\varphi_{22}\right)\end{array}\right], \quad g_{1}=\left[\begin{array}{c}-1 \\ 0\end{array}\right] g_{2}=\left[\begin{array}{l}1 \\ 0\end{array}\right]$ 


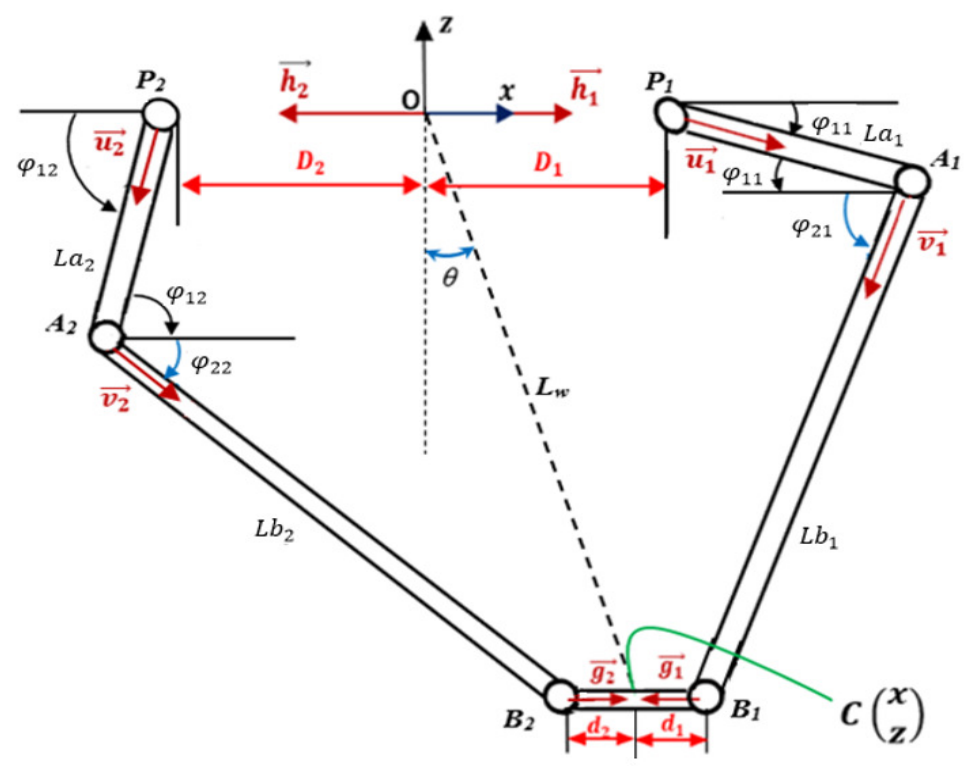

Figure 4: Parameterization of the PAR2 robot.

After differentiation of equation (25), we get:

$$
\delta \overrightarrow{O C}=\delta D_{i} \overrightarrow{h_{i}}+\delta L a_{i} \overrightarrow{u_{i}}+L a_{i} \delta \varphi_{1 i} E_{1 i} \overrightarrow{u_{i}}+\delta L b_{i} \overrightarrow{v_{i}}+L b_{i} \delta \varphi_{2 i} E_{2 i} \overrightarrow{v_{i}}+\delta d_{i} \overrightarrow{g_{i}}
$$

The matrices $E_{1 i}$ and $E_{2 i}$ are defined by:

$$
E_{11}=E_{22}=\left[\begin{array}{cc}
0 & 1 \\
-1 & 0
\end{array}\right]
$$

and

$$
E_{12}=E_{21}=\left[\begin{array}{cc}
0 & -1 \\
1 & 0
\end{array}\right]
$$

where $\delta O C$ is the position error of the manipulator. Similarly, $\delta D_{i}, \delta L a_{i}, \delta L b_{i}, \delta d_{i}, \delta \varphi_{1 i}$ and $\delta \varphi_{2 i}$ denote the variations of $D_{i}, L a_{i}, L b_{i}, d_{i}, \varphi_{1 i}$ and $\varphi_{2 i}$, respectively.

Equation (26) can be expressed in matrix form as follows:

$$
\delta O C=J \delta P
$$

such that:

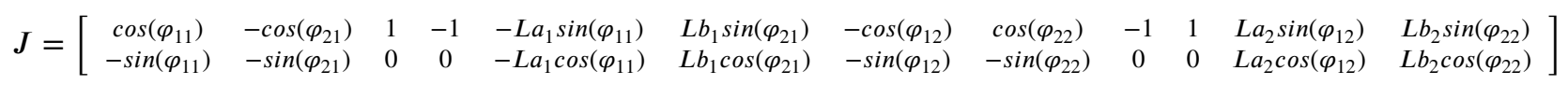

and:

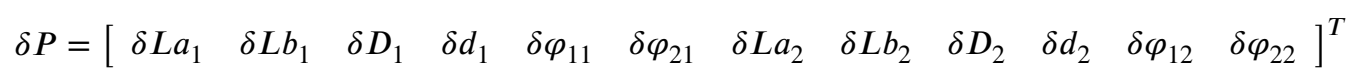


To calculate the $L S I$ of the variation in the dimensions, it is necessary to know the articular variables $\varphi_{1 i}$ and $\varphi_{2 i}$. For this purpose, a database of these variables must be available. The kinematic equation of chain 1 of the PAR 2 robot is given by the following relation [36]:

$$
L_{W}=\|\overrightarrow{O C}\|=\sqrt{\left(\frac{D-d}{2}\right)^{2}+L_{a}^{2}+L_{b}^{2}-2 L_{a} L_{b} \cos \left(\varphi_{11}+\varphi_{21}\right)+(D-d)\left(L_{a} \cos \left(\varphi_{11}\right)-L_{b} \cos \left(\varphi_{21}\right)\right)}
$$

where:

$L_{W} \longrightarrow L_{W_{\min }}$ if: $\varphi_{11}+\varphi_{21} \longrightarrow \frac{\pi}{6}$

$L_{W} \longrightarrow L_{W_{\max }}$ if: $\varphi_{11}+\varphi_{21} \longrightarrow \frac{11 \pi}{12}$

where:

$\frac{19 \pi}{12} \leq \varphi_{11} \leq 2 \pi$

From the calculation of the active joint variables $\varphi_{11}$ and $\varphi_{21}$, we can find the coordinates of the effector from the resolution of the following system of equation for chain 1 :

$$
\left\{\begin{array}{l}
C_{x}=L a_{1} \cos \left(\varphi_{11}\right)-L b_{1} \cos \left(\varphi_{21}\right)+D_{1}-d_{1} \\
C_{z}=-L a_{1} \sin \left(\varphi_{11}\right)-L b_{1} \sin \left(\varphi_{21}\right)
\end{array}\right.
$$

The values of $C_{x}$ and $C_{z}$ are used to solve the following system of equations for chain 2:

$$
\left\{\begin{array}{l}
C_{x}=L a_{2} \cos \left(\varphi_{12}\right)+L b_{2} \cos \left(\varphi_{22}\right)-D_{2}+d_{2} \\
C_{z}=-L a_{2} \sin \left(\varphi_{12}\right)-L b_{2} \sin \left(\varphi_{22}\right)
\end{array}\right.
$$

The resolution of this system makes it possible to find the passive articular variables $\varphi_{12}$ and $\varphi_{22}$. This procedure provides a database of active $\left(\varphi_{1 i}\right)$ and passive $\left(\varphi_{2 i}\right)$ joints variables, $i=1, \cdots, 2$. These variables are injected into the Jacobian matrix $J$ (expression (30)) to find the $L S I$ of each dimensional variation.

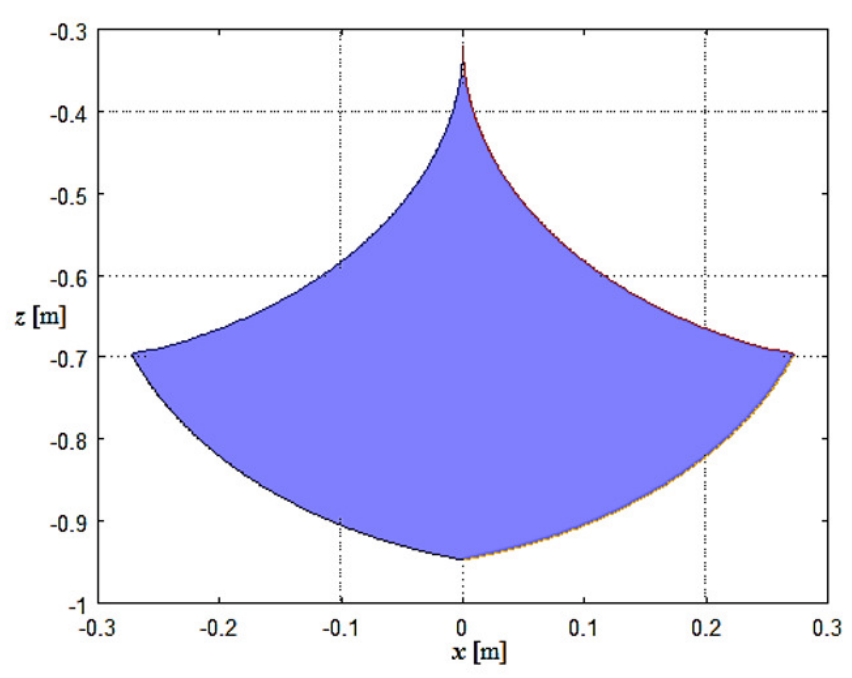

Figure 5: PAR2 PKM Workspace $W$.

\subsection{Sensitivity analysis of the PAR2 manipulator}

The aim of sensitivity analysis is to discover the significant error components among all the sources of error. To examine the error source that can trigger a greater error for the PAR2 manipulator end-effector, we can define all the error sources at the same level as one category. For this purpose, the error sources are classified into two groups 
according to their units (as shown in equation (10)).

In this paper, the error sensitivity indices $S I, G S I$ and $V S I$ are defined to reveal the influence of an error component on the position error of the manipulator so that the main error sources can be reasonably selected. These sensitivity indices have a clear physical significance and the theory shows that they are dimensionless (as shown in equation (9)). We assume that the manipulator end-effector operates in the workspace shown in Figure 5. Based on equation (23), and when only the linear error sources are taken into account, the $G S I s$ of the linear error sources on the position error of the end-effector are as shown in Figure 6. It can be seen from Figure 6, that the GSIs of the dimensional variations $\delta D_{i}(x), \delta L a_{i}(x), \delta L b_{i}(x)$, and $\delta d_{i}(x), i=1, \cdots, 2$, cancel each other, and there remains only the effect of the $G S I$ of the dimensional variations $\delta L a_{i}(z)$, and $\delta L b_{i}(z)$, because of the symmetry of the manipulator with respect to the $z$ axis.

The largest $G S I$ is that of $\delta L a_{i}(z)$ and $\delta L b_{i}(z)$, which means that these error have a greater average influence on the position errors of the manipulator than all the other geometric errors. Therefore, $\delta L a_{i}(z)$ and $\delta L b_{i}$ are the most important errors and must be taken into consideration during the design process. In contrast, the components of the linear errors $\delta D_{i}(z)$ and $\delta d_{i}(z)$ have no influence on the position error.

Similarly, based on the same equation (equation (23)), and when only the angular errors are taken into account, the

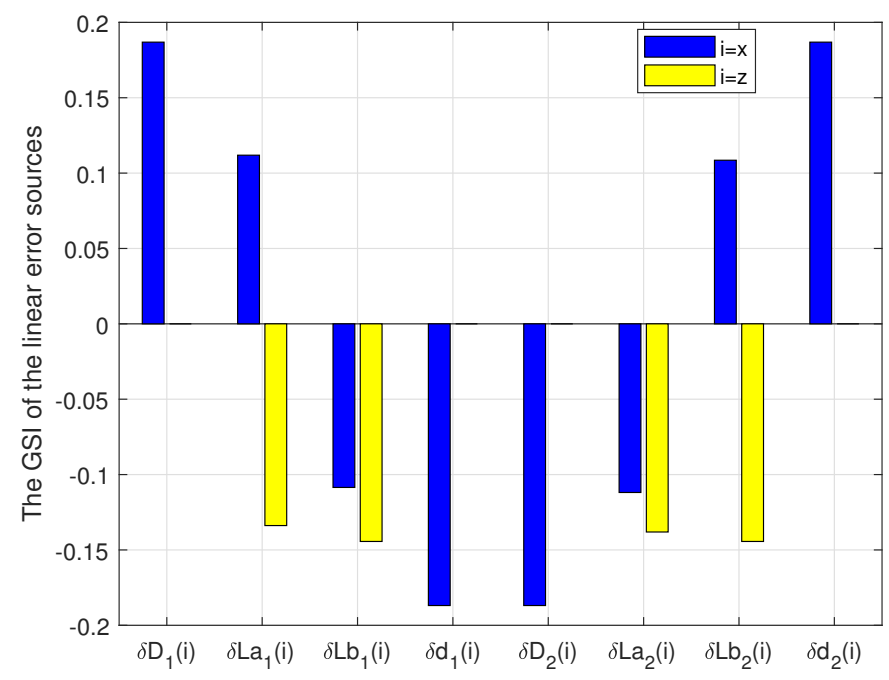

Figure 6: The GSI of the linear error sources on the position error.

$G S I$ of the angular errors sources on the position of the end-effector are illustrated in Figure 7. It can be seen in Figure 7 that; the $G S I s$ of the angular variations $\delta \varphi_{1 i}(x), \delta \varphi_{2 i}(x), i=1, \cdots, 2$, cancel each other out, and the angular error sources in the $z$ direction, $\delta \varphi_{2 i}(z)$ have a greater average influence on the position error of the manipulator than the other error sources.

According to the analysis in the above subsections (subsections 2.3 and 2.4), it is not only the average influence of an error component that is important for the accuracy of the end-effector, but also the variance of the influence of an error component around its average influence in the entire workspace. This variance significantly affects the accuracy of the end-effector. Using equation (24), the VSIs of the linear error component on the end-effector position error are shown in Figure 8. According to Figure $8, \delta D_{i}(x)$ and $\delta d_{i}(x)$ have the largest VSI, which indicates that the fluctuation of the LSI around the VSI is greater than for the other sources of error. The VSI of the angular error components on the position error of the end-effector are shown in Figure 9. We can note from Figure 9 that the effects of the angular error components $\varphi_{2 i}(x), i=1, \cdots, 2$, vary considerably. These angular error components require more consideration in the design stage. This information can also be used for a robust design. That is, if precision is required, then in the design phase, we can choose the most influencing parameter. In this case, the $L b_{i}$ segments can be manufactured with tight tolerances, while all the other segments are manufactured with wide tolerances. In this case, we can obtain a robust 


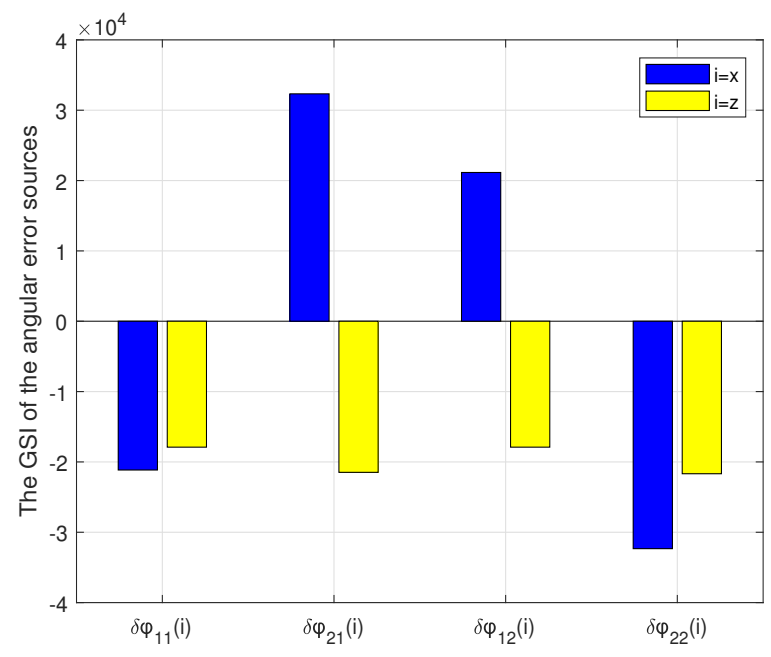

Figure 7: The GSI of the angular error sources on the position error.

mechanism, which meets the requirements mentioned in the specifications (precision in our case), with a minimum price.

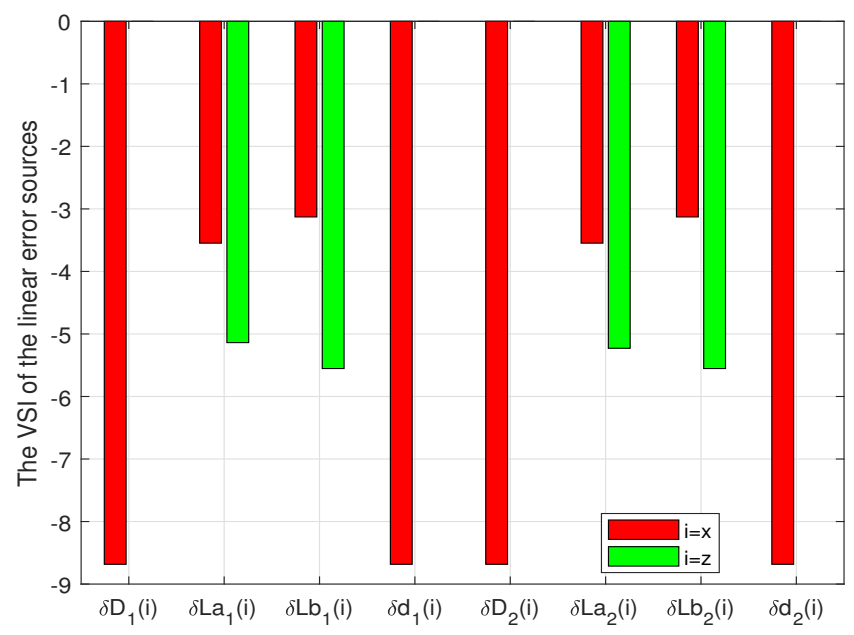

Figure 8: The VSI of the linear error sources on the position error.

\subsection{Verification of the sensitivity analysis of the position error}

After the sensitivity analysis has been performed on the PAR2 PKM as in section 3, the main sources of error can be found. The effect of these sources can be verified graphically in Figures 10, 11 and 12.

The linear error components $\delta L b_{i}$, can be identified as the main sources of the linear errors in the position error of the manipulator end-effector, while the angular error sources $\delta \varphi_{2 i}$ can be identified as the main sources of angular errors in the position error of the manipulator end-effector.

The identified main sources of errors have a much greater influence on manipulator accuracy than any other error 


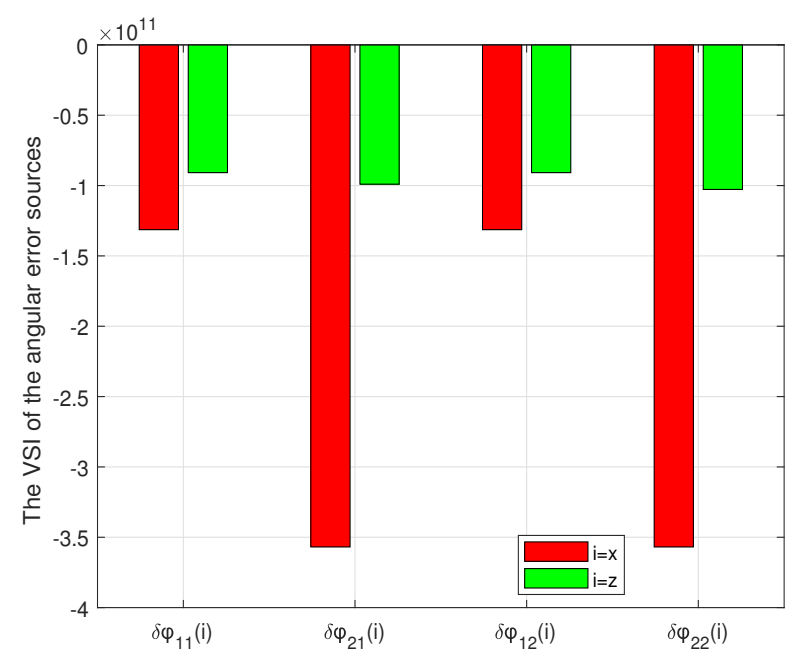

Figure 9: The VSI of the angular error sources on the position error.

component. It is not easy to compensate for these error components, which must be taken into account in the design phase. Therefore, and based on the proposed method for error sensitivity analysis, numerical simulation provides a suitable approach for testing the validity of the precision design.

To test the position errors under different conditions, we select thirteen representative points distributed in the manipulator workspace, as shown in Figure 13. From equation (29) the position error is first calculated with a variation of the linear error sources equal to $0.1 \mathrm{~mm}$ and with a variation of the angular error sources equal to $0.1^{\circ}$. The results of the calculation are shown in Figures 10, 11, and 12 (blue curves). In the precision design of the manipulator, the main sources of linear errors $\delta L b_{i}, i=1, \cdots, 2$, are reduced from $0.1 \mathrm{~mm}$ to $0.01 \mathrm{~mm}$, and the main sources of angular errors $\delta \varphi_{2 i}, i=1, \cdots, 2$, are reduced from $0.1^{\circ}$ to $0.01^{\circ}$.

The variations of the position error as a function of the variations of the main errors are illustrated in Figures 10, 11, and 12. From these figures, it appears that the effect of the main angular errors (the green and red curves in Figure 11 ) is greater than the effect of the main linear errors (the green and red curves in Figure 10). Also, it seems that the manipulator accuracy is at its maximum when the end-effector is in the P3 situation, that is, in the configuration with: full extension on the $z$-axis.

Finally, the average position error of the end-effector for the thirteen points is calculated for both cases, without and with precision design. The compared results are illustrated in Figure 14.

Based on our sensitivity analysis method, the four main sources of error $\left(\delta L b_{1}, \delta L b_{2}, \delta \varphi_{21}\right.$ and $\left.\delta \varphi_{22}\right)$ are improved by $10 \%$ over their original values to increase the accuracy of the manipulator. We can conclude, according to Figure 14 , that if we only improve the accuracy of the four main errors, this represents $33.3 \%$ of the total error components (12 errors). The position error will be improved from 2.61 to $1.257 \mathrm{~mm}$, indicating that the manipulator accuracy will be increased by $51.8 \%$. If we improve the accuracy of all the errors that are not vital errors, which account for $66.6 \%$ of the total error components, the position error will be improved from 2.61 to $1.614 \mathrm{~mm}$, indicating that the robot accuracy will be increased by $38.16 \%$. These results indicate that the error sensitivity analysis method and indices proposed in this paper are very effective.

It is worth to highlight that if the vital error has a significant effect on the sensitivity of the robot, we will have a considerable improvement on the precision of the robot if this error is reduced. In the case of the PAR2 robot, the improvement in the obtained accuracy is about $51.8 \%$, which indicates that the vital errors do not have a significant effect on the robot position error. 


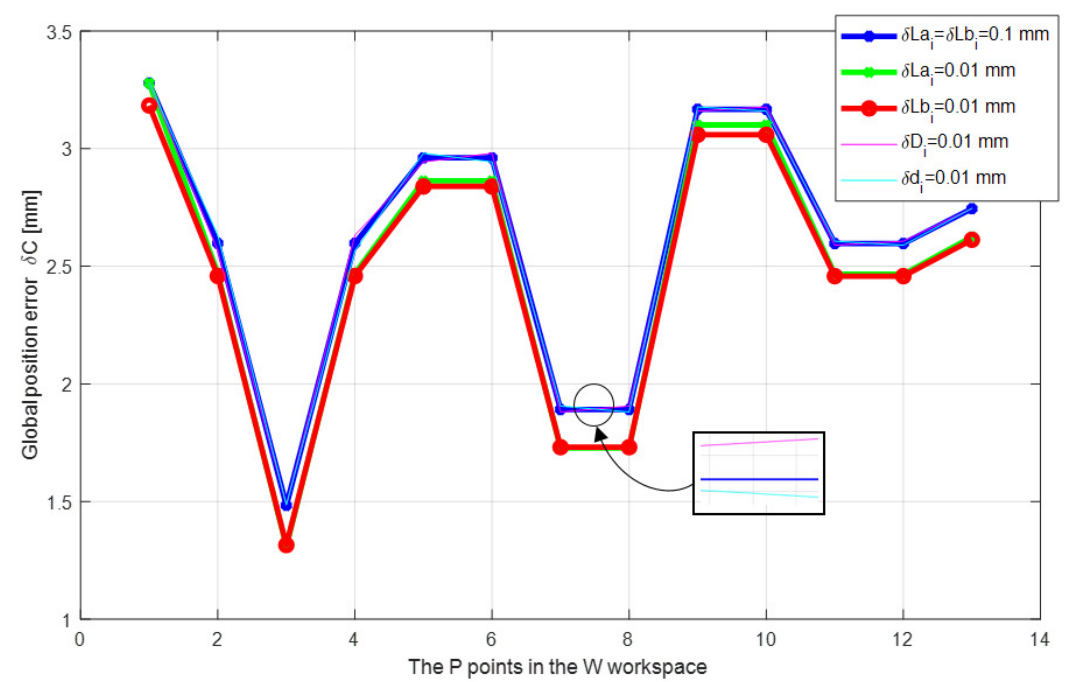

Figure 10: Variation of the position error as a function of the variation of linear errors.

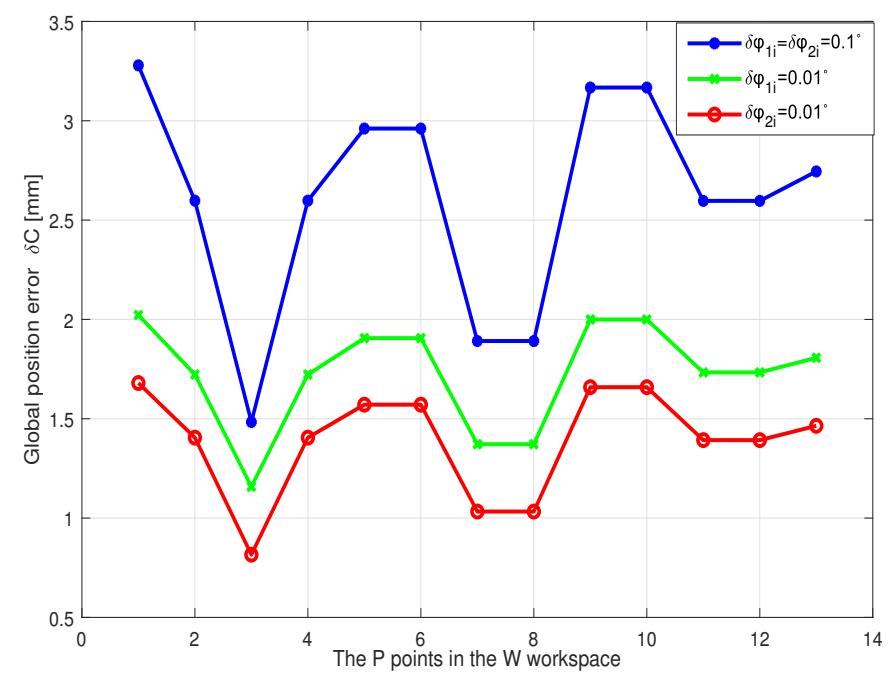

Figure 11: Variation of the position error as a function of the variation of angular errors.

\section{Conclusion and future work}

In this paper, a relationship between the sources of the geometric errors and the position errors of the PAR2 PKM end-effector is established by deriving the position vector. Based on the expression for the sensitivity coefficient, a sensitivity index $(S I)$ for the linear and angular error components is established. This index can accurately reveal the contribution of the error sources to the manipulator position errors for a specific pose. The index $(G S I)$ reflects the average influence of errors in a given workspace. The variance $(V S I)$ reflects the fluctuation of the $S I$ around the $G S I$ in the given workspace. Error sensitivity analysis is then performed using the $G S I$ and the $V S I$. Based on the numerical simulation of the error sensitivity analysis, the precision design of linear and angular errors is carried out. The results show that $33.3 \%$ of the error components (main errors) among all error sources can be improved to achieve a $51.8 \%$ improvement in the accuracy of the position error. On the other hand, if we improve the accuracy of 


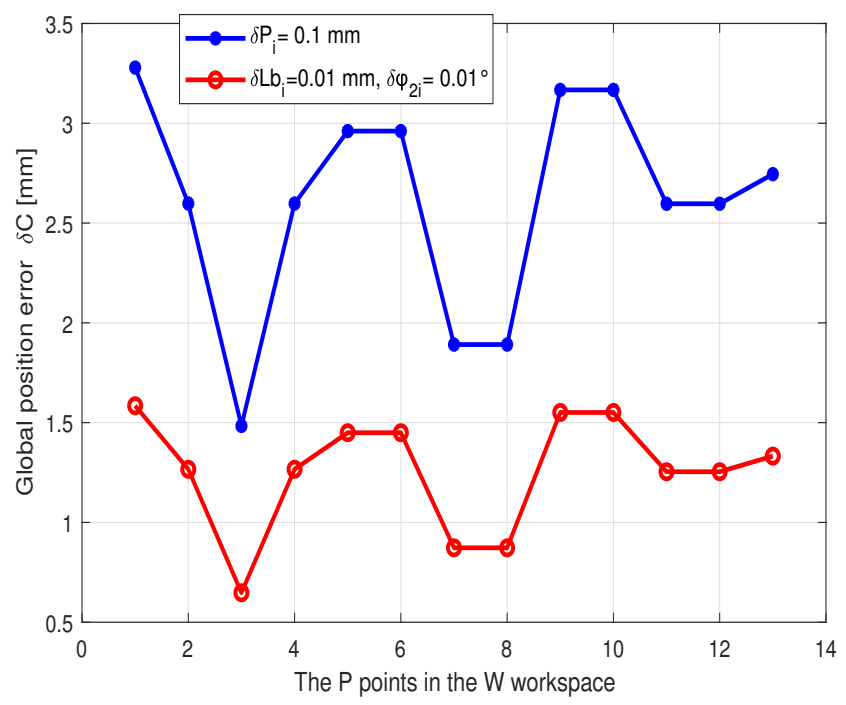

Figure 12: Variation of the position error as a function of the variation of main errors.

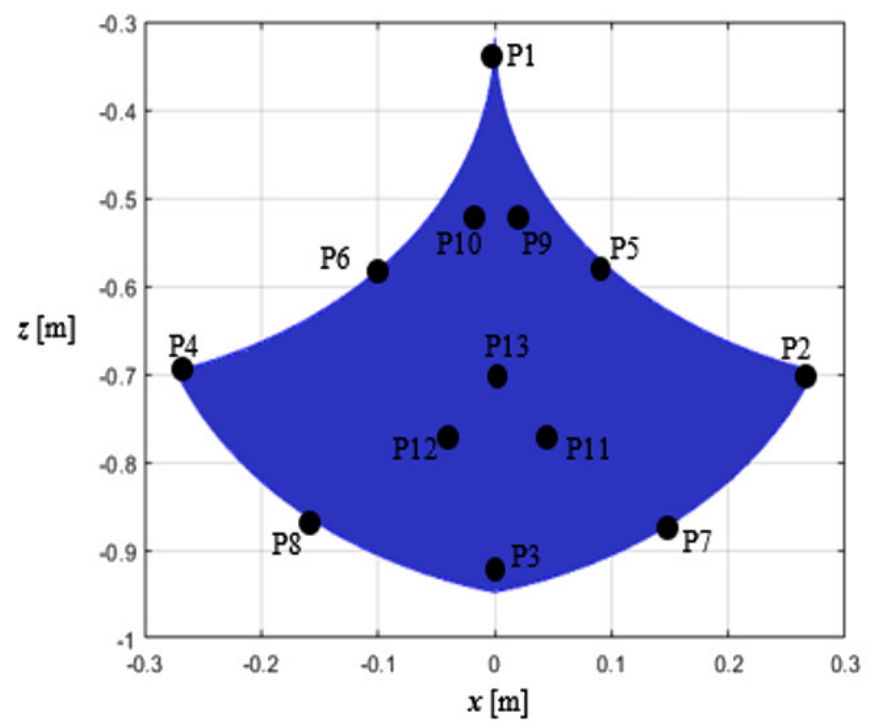

Figure 13: The Pi points in the $W$ workspace of the PAR2 PKM.

all the non-main errors, which account for $66.6 \%$ of the total error components, the position error is only improved by $38.16 \%$. These results indicate that the error sensitivity analysis method is quite effective, and can greatly improve the effects of precision design. In addition, in order to obtain a high accuracy at the design stage, the error components $\delta L b_{1}, \delta L b_{2}, \delta \varphi_{21}$ and $\delta \varphi_{22}$ must be given much greater consideration.

The problem addressed in this paper is the development of a dimensionless sensitivity index and its application on a PAR2 robot, the results obtained show the effectiveness of the developed index to determine the vital errors. As future work, we will work on the application of this index on different parallel robots with spatial structure such as the Gouth-Stewart PKM, and then study and experimentally measure the effect of this index on the improvement of the accuracy of a parallel robot. 


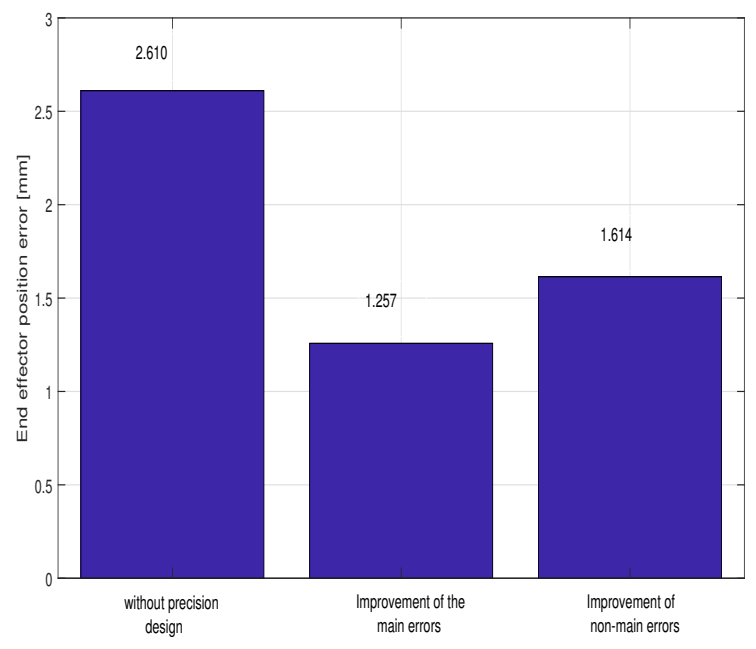

Figure 14: Comparison between design with and without precision.

\section{Acknowledgment}

This research was supported by the Directorate General for Scientific Research and Technological Development (DGRSDT) in Algeria.

\section{References}

[1] A. Verl, A. Valente, S. Melkote, C. Brecher, E. Ozturk, L. T. Tunc, Robots in machining, CIRP Annals 68 (2) (2019) 799 - 822. doi: 10.1016/j.cirp.2019.05.009.

[2] K. E. Zanganeh, J. Angeles, Kinematic isotropy and the optimum design of parallel manipulators, The International Journal of Robotics Research 16 (2) (1997) 185-197. doi:10.1177/027836499701600205.

[3] C. M. Gosselin, The optimum design of robotic manipulators using dexterity indices, Robotics and Autonomous Systems 9 (4) (1992) 213 226. doi:10.1016/0921-8890(92)90039-2.

[4] J. Wang, X.-J. Liu, Analysis of a novel cylindrical 3-dof parallel robot, Robotics and Autonomous Systems 42 (1) (2003) 31 - 46. doi: 10.1016/S0921-8890(02)00296-8.

[5] O. Company, Machines-outils rapides a structure parallele, méthodologie de conception, applications et nouveaux concepts, Ph.D. thesis, Université de MONTPELLIER II (2000).

[6] R. Kelaiaia, Improving the pose accuracy of the delta robot in machining operations., Int J Adv Manuf Technol 91 (2017) 2205-2215. doi: 10.1007/s00170-016-9955-8.

[7] M. Weck, D. Staimer, Parallel kinematic machine tools - current state and future potentials, CIRP Annals 51 (2) 671 - 683. doi: 10.1016/ S0007-8506(07)61706-5.

[8] A. Patel, K. Ehmann, Volumetric error analysis of a stewart platform-based machine tool, CIRP Annals 46 (1) (1997) 287 - 290. doi: 10.1016/S0007-8506(07)60827-0.

[9] T. Huang, Y. Li, G. Tang, S. Li, X. Zhao, J. WhitehouseDavid., G. ChetewynDerek, L. Xian-ping, Error modeling, sensitivity analysis and assembly process of a class of 3-dof parallel kinematic machines with parallelogram struts, Science in China Series E: Technological Science 45 (2002) 467-476.

[10] G. J. Shijie Guo, Xuesong Mei, Geometric accuracy enhancement of five-axis machine tool based on error analysis, Int J Adv Manuf Technol 105 (2019) 137-153. do i:10.1007/s00170-019-04030-4.

[11] F. X. Jie Li, X.-J. Liu, Geometric error modeling and sensitivity analysis of a five-axis machine tool, Int J Adv Manuf Technol 82 (2016) 2037-2051. doi:10.1007/s00170-015-7492-5.

[12] F. X. Jie Li, X.-J. Liu, A spatial vector projection based error sensitivity analysis method for industrial robots, Journal of Mechanical Science and Technology 32 (6) (2016) 2839-2850. doi:10.1007/s12206-018-0540-y.

[13] Y. J. H. L. J. Z. Qingzhao Li, Wei Wang, Z. Jiang, A sensitivity method to analyze the volumetric error of five-axis machine tool., Int J Adv Manuf Technol 98 (2018) 1791-1805. doi:10.1007/s00170-018-2322-1.

[14] Q. Z. Sungcheul Lee, K. F. Ehmann, Error modeling for sensitivity analysis and calibration of the tri-pyramid parallel robot, Int J Adv Manuf Technol 93 (2017) 1319-1332. doi:10.1007/s00170-017-0590-9. 
[15] K.-C. Fan, H. Wang, J.-W. Zhao, T.-H. Chang, Sensitivity analysis of the 3-prs parallel kinematic spindle platform of a serial-parallel machine tool, International Journal of Machine Tools and Manufacture 43 (15) (2003) 1561- 1569. do i:10.1016/S0890-6955(03)00202-5.

[16] S. Caro, P. Wenger, F. Bennis, D. Chablat, Sensitivity analysis of the orthoglide: A three-dof translational parallel kinematic machine, Journal of Mechanical Design 128 (2) (2005) 392-402. doi:10.1115/1.2166852.

[17] D. Li, P. Feng, J. Zhang, D. Yu, Z. Wu, An identification method for key geometric errors of machine tool based on matrix differential and experimental test, Proceedings of the Institution of Mechanical Engineers, Part C: Journal of Mechanical Engineering Science 228 (17) (2014) 3141-3155. doi:10.1177/0954406214527272.

[18] P. W. Stephane Caro, Nicolas Binaud, Sensitivity analysis of 3-rpr planar parallel manipulators, Journal of Mechanical Design 131 (12). doi: $10.1115 / 1.4000216$.

[19] M. Tannous, S. Caro, A. Goldsztejn, Sensitivity analysis of parallel manipulators using an interval linearization method, Mechanism and Machine Theory 71 (2014) 93 - 114. doi:10.1016/j . mechmachtheory .2013.09.004.

[20] L. C. Matteo Palpacelli, Giacomo Palmieri, D. Corinaldi, Sensitivity analysis and model validation of a 2-dof mini spherical robot., Intell Robot Syst 91 (2017) 155-163. doi:10.1007/s 10846-017-0679-2.

[21] Y. W. C. Y. Lizhe Qi, Leibin, Analysis of the robot positioning error based on jacobian matrix, in: D. Jin and S. Lin (Eds): Advances in Mechanical and Electronic Engineering, Vol. 176, LNEE, 2012, pp. 329-334. doi : 10.1007/978-3-642-31507-7_54.

[22] X. H. Zhang, P. Ba, L. Mu, Position error sensitivity analysis for polishing robot, in: Advances in Materials Processing X, Vol. 500 of Advanced Materials Research, Trans Tech Publications Ltd, 2012, pp. 326-330. doi:10.4028/www.scientific.net/AMR.500.326.

[23] Z. Li, W. Feng, J. Yang, Y. Huang, An investigation on modeling and compensation of synthetic geometric errors on large machine tools based on moving least squares method, Proceedings of the Institution of Mechanical Engineers, Part B: Journal of Engineering Manufacture 232 (3) (2018) 412-427. doi:10.1177/0954405416645985.

[24] H. Chanal, E. Duc, J. Hascoët, P. Ray, Reduction of a parallel kinematics machine tool inverse kinematics model with regard to machining behaviour, Mechanism and Machine Theory 44 (7) (2009) 1371-1385. doi:https ://doi .org/10.1016/j.mechmachtheory . 2008.11. 004.

[25] X. M. Shijie Guo, Gedong Jiang, Investigation of sensitivity analysis and compensation parameter optimization of geometric error for five-axis machine tool, Int J Adv Manuf Technol 93 (2017) 3229-3243. doi:10.1007/s00170-017-07556.

[26] Y. Ni, C. Shao, B. Zhang, W. Guo, Error modeling and tolerance design of a parallel manipulator with full-circle rotation, Advances in Mechanical Engineering 8 (5) (2016) 1687814016649300. doi:10.1177/1687814016649300.

[27] L. X. Chen Y., Xie F., Z. Y., Error modeling and sensitivity analysis of a parallel robot with scara (selective compliance assembly robot arm) motions, Chinese Journal of Mechanical Engineering 27. doi: 10.3901/CJME. 2014.0423.082.

[28] G. Z. P. G. Qiang Cheng, Hongwei Zhao, L. Cai, An analytical approach for crucial geometric errors identification of multi-axis machine tool based on global sensitivity analysis., Int J Adv Manuf Technol 75 (2014) 107-121. doi:10.1007/s00170-014-6133-8.

[29] S.-R. G. Gang Cheng, J.-L. Yu, Sensitivity analysis and kinematic calibration of 3-ucr symmetrical parallel robot leg, Journal of Mechanical Science and Technology 25 (7) (2011) 647-1655. doi:10.1007/s12206-011-0417-9.

[30] O. Rodrigues, Des lois géométriques qui régissent les déplacements d'un système solide dans l'espace, et de la variation des coordonnées provenant de ces déplacements considérés indépendamment des causes qui peuvent les produire, Journal de mathématiques pures et appliquées 5 (1) (1840) 380-440.

[31] C. W. Tian Wenjie, Gao Weiguo, Y. Nie, Error modeling and sensitivity analysis of a five-axis machine tool, Mathematical Problems in Engineering 2014 (2014) 1-8. doi: 10.1155/2014/745250.

[32] A. Brahmia, R. Kelaiaia, A. Chemori, O. Company, On Robust Mechanical Design of a PAR2 Delta-Like Parallel Kinematic Manipulator, Journal of Mechanisms and Robotics 14 (1). doi:10.1115/1.4051360.

[33] J. Jeong, Kinematic calibration for redundantly actuated parallel mechanisms, Ph.D. thesis, School of Mechanical and Aerospace Engineering, Seoul National University (2002).

[34] G. S. Natal, A. Chemori, P. Francois, Nonlinear control of parallel manipulators for very high accelerations without velocity measurement: stability analysis and experiments on par2 parallel manipulator, Robotica 34 (1) (2016) 43-70. doi: 10.1017/S0263574714001246.

[35] O. Company, F. Pierrot, S. Krut, C. Baradat, V. Nabat, Par2: a spatial mechanism for fast planar two-degree-of-freedom pick-and-place applications., Meccanica 46 (2011) 239-248. doi:10.1007/s11012-010-9413-x.

[36] R. Kelaiaia, A. Zaatri, O. Company, L. Chikh, Some investigations into the optimal dimensional synthesis of parallel robots., Int J Adv Manuf Technol 83 (2016) 1525-1538. doi:10.1007/s00170-015-7611-3. 